\title{
Quark-Gluon Plasma with Finite Quark Mass
}

Bimal Kumar Majumdar

B. K. C. College, Calcutta 700035, India

Rajkumar Roychoudhury

Electronics Unit, Indian Statistical Institute, Calcutta 700035, India

Z. Naturforsch. 45a, 95-101 (1990); received January 27, 1989*

\begin{abstract}
We have derived exact analytical formulas of various thermodynamic quantities such as Energy, Pressure, Entropy etc., at finite temperature without neglecting quark mass. The critical curve in the $\mu-T$ plane has been calculated to show the effect of finite quark mass. The energy density has also been calculated for different values of the quark mass.
\end{abstract}

\section{Introduction}

The quark-gluon plasma may play an important role in understanding the strong interaction. Extensive studies [1] have been made in this direction. Some authors [1 k] have even argued that the creation of such a plasma in the laboratory can make the involved physics much clearer since one can then use the well established statistical physics and can thereby correlate the microscopic information with experimentally observable quantities.

Also the various aspects of confinement and deconfinement of quarks as well as the phase-transition from hadronic to quark matter have been studied [1] by many authors. The two phase character of strongly interacting matter has been discussed from the phenomenological point of view [2]. QCD gives rise to the expectation that the two-phase character and the phase-transition can be explained by the same basic theory [3], and developments in the lattice QCD at finite temperature [4-9] support this hope. Miller and Redlich [10] had recently discussed the thermodynamical properties of models with non-Abelian internal symmetries.

But in all previous works, the mass of the quarks has been neglected, arguing that in most cases $(u$ and $d$ quarks) it is negligible. But when heavy flavours are included, the quark mass cannot be neglected. For example the thermodynamic abundance of charm with a mass of about $1500 \mathrm{MeV}$, is low but is still significant [11] and bound to have an effect on thermodynamical quantities. Also, there is a quite apprecia-

\footnotetext{
* Revised version received 14. September, 1989.

Reprint requests to R. Roychoudhury, Electronics Unit, Indian Statistical Institute, Calcutta 700035, India.
}

ble $\mathrm{S}$-abundance, and the mass of the strange quark is not small enough (about $180 \mathrm{MeV}$ ) to be neglected.

We therefore have taken up calculations concerning quark gluon plasmas (on the basis of the bag model [11-15]), taking into account finite quark masses. It has been found that exact analytical formulas for various thermodynamical quantities, such as energy, pressure, entropy, etc. can be found without neglecting the quark masses or at least taking into account first order corrections. The expressions for the energy in the limit $T \rightarrow 0$ can easily be found, and this formula can be applied easily to quark bag models for predicting the mass spectrum of hadrons. In the present paper, our object is to show the effect of the quark masses, and we will discuss quantitatively its contribution to various thermodynamical quantities.

To make the relevant formulae most general, we have considered quantum statistical grand partition functions, i.e. $F-D$ for quarks and $B-E$ for gluons. It will be shown that it is possible to get exact analytical expressions for all the desired physical quantities with finite quark and gluon masses.

In Sect. 2, starting from grand partition functions, the mass formulae for hadrons at zero temperature are obtained. In Sect. 3, the thermodynamical quantities for the quark-gluon plasma are evaluated. Section 4 is devoted to the calculation of the critical temperature. In Sect. 5 we discuss phase transitions and the effect of finite quark mass on the energy density. The final section is kept for comments and discussions.

\section{Mass Formulae for Hadrons}

In this section we derive the mass formulae for hadrons with the help of the bag model [11-15]. To do

0932-0784 / 90 / 0200-0095 \$01.30/0. - Please order a reprint rather than making your own copy. 
this, we start from the thermodynamical potential for a free quark and gluon gas, for which we get the Helmholtz free energy. The thermodynamical potential in reduced units is given by

with

$$
\begin{array}{r}
\Omega_{\mathrm{f}}=-\frac{V}{2 \pi^{2} \beta} \int k^{2} \mathrm{~d} k\left[g _ { \mathrm { qu } } \sum _ { \alpha } \operatorname { l n } \left(1+\exp \left(\mu_{\alpha}-\varepsilon_{\alpha}\right) \beta\right.\right. \\
\left.-g_{\mathrm{glu}} \ln (1-\exp (\mu-\varepsilon) \beta)\right]
\end{array}
$$

$$
\varepsilon_{\alpha}=\left(k^{2}+m_{\alpha}^{2}\right)^{1 / 2} \text { and } \varepsilon=\left(k^{2}+m^{2}\right)^{1 / 2},
$$

where $\mu_{\alpha}$ and $m_{\alpha}$ are the chemical potential and mass of the $\alpha$ quark, $\mu$ and $m$ are the chemical potential and mass of the gluon, and $\beta$ is the inverse of temperature. $g_{\mathrm{gu}}\left(g_{\mathrm{glu}}\right)$ is the fraction of the quark (gluon) gas.

At zero temperature $(\beta \rightarrow \propto)$, since $\mu \rightarrow 0$, gluons will not contribute to $\Omega_{\mathrm{f}}$ and we have, as $T \rightarrow 0$ :

$$
\Omega_{\mathrm{f}}=-\frac{V g_{\mathrm{qu}}}{2 \pi^{2}} \sum_{\alpha}\left[I_{1}-I_{2}\right]
$$

with

$$
\begin{aligned}
& I_{1}=\int_{m_{\alpha}}^{\mu_{\alpha}} \mu_{\alpha} \varepsilon_{\alpha}\left(\varepsilon_{\alpha}^{2}-m_{\alpha}^{2}\right)^{1 / 2} \mathrm{~d} \varepsilon_{\alpha}, \\
& I_{2}=\int_{m_{\alpha}}^{\mu_{\alpha}} \varepsilon_{\alpha}^{2}\left(\varepsilon_{\alpha}^{2}-\mu_{\alpha}^{2}\right)^{1 / 2} \mathrm{~d} \varepsilon_{\alpha} .
\end{aligned}
$$

Evaluating these integrations we get

$$
\begin{aligned}
\Omega_{\mathrm{f}}=-\frac{V g_{\mathrm{qu}}}{2 \pi^{2}} \sum_{\alpha}[ & \frac{\mu_{\alpha}}{12}\left(\mu_{\alpha}^{2}-m_{\alpha}^{2}\right)^{3 / 2}-\frac{\mu_{\alpha} m_{\alpha}^{2}}{8}\left(\mu_{\alpha}^{2}-m_{\alpha}^{2}\right)^{1 / 2} \\
& \left.+\frac{m_{\alpha}^{4}}{8} \ln \left|\frac{\mu_{\alpha}+\left(\mu_{\alpha}^{2}-m_{\alpha}^{2}\right)^{1 / 2}}{m_{\alpha}}\right|\right]
\end{aligned}
$$

Using the standard relation between free energy and thermodynamic potential, the Helmholtz free energy $F_{\text {qu }}$ for free quarks is obtained as (for more details see [16])

$$
\begin{aligned}
F_{\mathrm{qu}}=\frac{V g_{\mathrm{qu}}}{2 \pi^{2}} \sum_{\alpha} & {\left[\frac{\mu_{\alpha}}{4}\left(\mu_{\alpha}^{2}-m_{\alpha}^{2}\right)^{3 / 2}+\frac{\mu_{\alpha} m_{\alpha}^{2}}{8}\left(\mu_{\alpha}^{2}-m_{\alpha}^{2}\right)^{1 / 2}\right.} \\
- & \left.\frac{m_{\alpha}^{4}}{8} \ln \left|\frac{\mu_{\alpha}+\left(\mu_{\alpha}^{2}-m_{\alpha}^{2}\right)^{1 / 2}}{m_{\alpha}}\right|\right],
\end{aligned}
$$

and the density of $\alpha$ quarks is

$$
\mathscr{S}_{\alpha}=\frac{g_{\mathrm{qu}}}{6 \pi^{2}}\left(\mu_{\alpha}^{2}-m_{\alpha}^{2}\right)^{3 / 2}
$$

Finally we get

$$
\begin{gathered}
F_{\mathrm{qu}}=g_{\mathrm{qu}} \sum_{\alpha}\left[\left(\frac{3}{4} N_{\alpha} V^{-1 / 3}+\frac{m_{\alpha}^{2}}{16 \pi^{2}} V^{1 / 3}\left(6 \pi^{2}\right)^{1 / 3}\right)\right. \\
\cdot\left(m_{\alpha}^{2} V^{2 / 3}+\left(6 \pi^{2}\right)^{2 / 3} N_{\alpha}^{2 / 3}\right)^{1 / 2}-\frac{m_{\alpha}^{2}}{16 \pi^{2}} V \\
\left.\cdot \ln \left|\frac{\left(\left(6 \pi^{2}\right)^{2 / 3} N_{\alpha}^{2 / 3} V^{-2 / 3}+m_{\alpha}^{2}\right)^{1 / 2}+\left(6 \pi^{2}\right)^{1 / 3} V^{-1 / 3}}{m_{\alpha}}\right|\right],
\end{gathered}
$$

where $N_{\alpha}=$ number of $\alpha$ quarks in the bag.

The one gluon exchange energy contributes to the total free energy of the bag. To get this, we used the color gluon exchange energy $-m R$ curve of De Grand et al. [12] and obtained by least squares fitting

$$
F_{\mathrm{gex}}=\sum_{\alpha} \frac{8}{3} \alpha_{\mathrm{s}} a_{\alpha}\left(A_{0} m_{\alpha}^{2} R-B_{0} m_{\alpha}+C_{0} R^{-1}\right),
$$

where $\alpha_{\mathrm{S}}$ is the coupling constant $=g^{2} / 4 \pi, R$ the radius of the bag and $a_{\alpha}$ the parameter for the $\alpha$ type (strange or non-strange) quark. Further

$$
A_{0}=0.00568, \quad B_{0}=0.0508, \quad C_{0}=0.1762 .
$$

Writing (2.10) in terms of the volume of the bag, we have

$F_{\text {gex }}=\sum_{\alpha} \frac{8}{3} \alpha_{\mathrm{S}} a_{\alpha}\left(A m_{\alpha}^{2} V^{1 / 3}-B_{0} m_{\alpha}+C V^{-1 / 3}\right)$,

where

$$
A=A_{0}\left(\frac{3}{4 \pi}\right)^{1 / 3} \text { and } C=C_{0}\left(\frac{4 \pi}{3}\right)^{1 / 3} .
$$

Now, since at zero temperature the free energy is equal to the internal energy, (2.11) may be considered as an expression for the free energy, $F_{\text {gex }}$. It is to be mentioned here that we have considered interactions between quarks of equal masses. To find the total free energy of the bag we should consider two more terms: surface and volume energy. They are given by

$$
F_{\mathrm{g}}=\sigma V^{2 / 3}, \quad F_{\mathrm{v}}=B V,
$$

where $\sigma$ and $B$ are free parameters. We have considered the surface energy as negative, following Kagiyama et al. [16]. At zero temperature, the free energy is equal to the internal energy. Thus we have

$$
\bar{E}=\left|F_{\mathrm{qu}}+F_{\mathrm{gex}}+F_{\mathrm{g}}+F_{\mathrm{v}}\right|_{T=0} .
$$

Again, the mass $M$ of the hadron is the minimum value of the internal energy. Therefore,

$$
M=\left.\bar{E}\right|_{V=\bar{V}} \text { with }\left.\quad \frac{\partial \bar{E}}{\partial V}\right|_{V=\bar{V}}=0 .
$$


Thus, with the help of (2.9), (2.11), (2.12), (2.13) and (2.14) we at once get the expression for $M$.

The mass of any hadron can be calculated from the expression for $M$, provided the values of the parameters $B, \sigma$ and $\alpha_{\mathrm{S}}$ are determined. The values of these parameters can be calculated by taking the masses of three hadrons. For $m_{\alpha}=0,(2.6),(2.7)$ and (2.8) become

$$
\begin{aligned}
& \Omega_{\mathrm{f}}=-\frac{V g_{\mathrm{qu}}}{24 \pi^{2}} \sum_{\alpha} \mu_{\alpha}^{4}, \\
& F_{\mathrm{qu}}=\frac{V g_{\mathrm{qu}}}{8 \pi^{2}} \sum_{\alpha} \mu^{4}, \\
& \mathscr{S}_{\alpha}=\frac{g}{6 \pi^{2}} \mu_{\alpha}^{3},
\end{aligned}
$$

these equations being consistent with those of Kagiyama et al. [16]. From the above expressions for the free energy of quarks it can be shown that the effect of the quark mass can not be neglected when quarks are heavy. A simple calculation shows that for $\mu=$ $400 \mathrm{MeV}$ and $m=100 \mathrm{MeV}$, the free energy changes by 9 percent compared to that of quarks with no mass. We have not attempted here to give an other fit to the mass spectrum, as it is highly sensitive to the values of $\sigma$ and $\alpha_{\mathrm{S}}$ and, as pointed out by Chin [1 e], a large value of $\alpha_{\mathrm{S}}$ (which is needed to explain hte masses of $\pi$ and $p$ ) will make the perturbative calculations not very reliable. However, one finds that the reasonable values $\alpha_{\mathrm{S}}=0.55$ and $B=145 \mathrm{Mev}$ will need $\sigma=-0.41$ to get the correct $\pi$ mass.

\section{Quark-gluon Plasma at $T \neq 0$}

Here we evaluate the various thermodynamical quantities of the quark-gluon plasma in vacuum. Vacuum means a region which exerts a pressure on the surface within which plasma is confined. To obtain the desired physical entities we use the grand canonical quantum statistical partition functions and the standard relations of the derived variables. Masses of quarks and gluons will be included in our calculations. The total energy of the system consists mainly of contributions from quarks, gluons and the vacuum or Bag term. In addition to this, we shall consider second order gluon-gluon interactions and interactions between quarks themselves via colour gluons. In this regard, only first order running coupling will be considered, viz., $\alpha_{\mathrm{S}}\left(q^{2}\right)=g^{2} / 4 \pi$ (Rafelski [1 k], Chin [1 e]). To find the vacuum contribution we introduce, like others, a vacuum partition function

$$
[\ln Z]_{\mathrm{vac}}=-B \beta V
$$

which ensures the positive energy density $B$ of confined quarks and gluons and a negative pressure on the surface of this region. Now, starting from the free quarks partition function, we have

$E_{\mathrm{qu}}=$ Energy density of the free quarks

$$
\begin{aligned}
& =-\frac{\partial}{\partial \beta}[\ln Z]_{\mathrm{qu}} \\
& =-\frac{\partial}{\partial \beta}\left[\frac{g_{\mathrm{qu}}}{2 \pi^{2}} \int_{m_{\alpha}}^{\alpha} \varepsilon_{\alpha}\left(\varepsilon_{\alpha}^{2}-m_{\alpha}^{2}\right)^{1 / 2} \ln \left[1+e^{\beta\left(\mu_{\alpha}-\varepsilon\right)}\right] \mathrm{d} \varepsilon\right] .
\end{aligned}
$$

This can be written as

$$
E_{\mathrm{qu}}=\frac{g_{\mathrm{qu}}}{2 \pi^{2} \beta^{4}} \int_{m_{\alpha} \beta}^{\alpha} \frac{x^{2}\left(x^{2}-m_{\alpha}^{2} \beta^{2}\right)^{1 / 2} \mathrm{~d} x}{\lambda^{-1} e^{x}+1},
$$

where $\lambda=\exp \left(\mu_{\alpha} \beta\right)$ and $x=\varepsilon \beta$.

$$
I=\int_{m_{\alpha} \beta}^{\infty} \frac{x^{2}\left(x^{2}-m_{\alpha}^{2} \beta^{2}\right)^{1 / 2}}{\lambda^{-1} e^{x}+1} \mathrm{~d} x=I_{1}-I_{2}+I_{3},
$$

(where we have assumed $\mu_{\alpha}>m_{\alpha}$. A separate calculation has been made when $\mu_{\alpha}=0$.), where

$$
\begin{aligned}
& I_{1}=\int_{m_{\alpha} \beta}^{\xi} x^{2}\left(x^{2}-m_{\alpha}^{2} \beta^{2}\right)^{1 / 2} \mathrm{~d} x, \\
& I_{2}=\int_{m_{\alpha} \beta}^{\xi} \frac{x^{2}\left(x^{2}-m_{\alpha}^{2} \beta^{2}\right)^{1 / 2} \mathrm{~d} x}{e^{\xi-x}+1}, \\
& I_{3}=\int_{\xi}^{\propto} \frac{x^{2}\left(x^{2}-m_{\alpha}^{2} \beta^{2}\right)^{1 / 2} \mathrm{~d} x}{e^{x-\xi}+1}
\end{aligned}
$$

with $\xi=\mu_{\alpha} \beta$.

Evaluating the integrals using standard techniques to handle Fermi integrals [17], we have

$$
\begin{aligned}
I_{1}=\frac{\beta^{4}}{4} & {\left[\mu_{\alpha}\left(\mu_{\alpha}^{2}-m_{\alpha}^{2}\right)^{3 / 2}+\frac{m_{\alpha}^{2}}{2}\left(\mu_{\alpha}\left(\mu_{\alpha}^{2}-m_{\alpha}^{2}\right)^{1 / 2}\right)\right.} \\
& \left.-\frac{m_{\alpha}^{4}}{8} \ln \left|\frac{\mu_{\alpha}+\left(\mu_{\alpha}^{2}-m_{\alpha}^{2}\right)^{1 / 2}}{m_{\alpha}}\right|\right] \\
I_{3}-I_{2}= & \pi^{2} \beta^{2}\left(\frac{\mu_{\alpha}^{2}}{2}-\frac{m_{\alpha}^{2}}{12}\right)+\pi^{4} \frac{7}{60} .
\end{aligned}
$$

Finally we get,

$$
\begin{aligned}
E_{\mathrm{qu}}=\frac{g_{\mathrm{qu}}}{2 \pi^{2}}[ & \frac{\mu_{\alpha}}{4}\left(\mu_{\alpha}^{2}-m_{\alpha}^{2}\right)^{3 / 2}+\frac{m_{\alpha}^{2}}{8}\left(\mu_{\alpha}\left(\mu_{\alpha}^{2}-m_{\alpha}^{2}\right)^{1 / 2}\right) \\
& -\frac{m_{\alpha}^{4}}{8} \ln \left|\frac{\mu_{\alpha}+\left(\mu_{\alpha}^{2}-m_{\alpha}^{2}\right)^{1 / 2}}{m_{\alpha}}\right| \\
& \left.+\left(\frac{\mu_{\alpha}^{2}}{2}-\frac{m_{\alpha}^{2}}{12}\right)(\pi T)^{2}+\frac{7}{60}(\pi T)^{4}\right]
\end{aligned}
$$


Now since each quark has the baryon number $\frac{1}{3}$, we have $3 \mu_{\alpha}=\mu$. Therefore

$$
\begin{aligned}
E_{\mathrm{qu}}=\frac{g_{\mathrm{qu}}}{2 \pi^{2}}[ & \frac{1}{4}\left(\frac{\mu}{3}\right)\left(\left(\frac{\mu}{3}\right)^{2}-m_{\alpha}^{2}\right)^{3 / 2} \\
& +\frac{m_{\alpha}^{2}}{8}\left(\left(\frac{\mu}{3}\right)\left(\left(\frac{\mu}{3}\right)^{2}-m_{\alpha}^{2}\right)^{1 / 2}\right. \\
& -\frac{m_{\alpha}^{4}}{8} \ln \left|\frac{\left(\frac{\mu}{3}\right)+\left(\left(\frac{\mu}{3}\right)^{2}-m_{\alpha}^{2}\right)^{1 / 2}}{m_{\alpha}}\right| \\
& \left.+\left(\frac{1}{2}\left(\frac{\mu}{3}\right)^{2}-\frac{m_{\alpha}^{2}}{12}\right)(\pi T)^{2}+\frac{7}{60}(\pi T)^{4}\right] .
\end{aligned}
$$

Similarly, from the corresponding partition function we get the free gluon energy density $E_{\text {glu }}$ as

$$
\begin{aligned}
E_{\mathrm{glu}} & =-\frac{\partial}{\partial \beta}(\ln Z)_{\mathrm{glu}}=\frac{g_{\mathrm{glu}}}{2 \pi^{2} \beta^{4}} \int_{m \beta}^{\propto} \frac{x^{2}\left(x^{2}-m^{2} \beta^{2}\right)^{1 / 2} \mathrm{~d} x}{\lambda^{-1} e^{x}-1} \\
& =\frac{g_{\mathrm{glu}}}{2 \pi^{2} \beta^{4}}\left(I_{1}^{\prime}+I_{2}^{\prime}\right) \quad \text { with } \quad x=\varepsilon \beta .
\end{aligned}
$$$$
I_{1}^{\prime}=\sum_{n=1}^{\infty} \int_{m \beta}^{\infty}\left(x^{2}-m^{2} \beta^{2}\right)^{3 / 2} e^{-n x} \mathrm{~d} x,
$$

$I_{2}^{\prime}=m^{2} \beta^{2} \sum_{n=1}^{\infty} \int_{m \beta}^{\infty}\left(x^{2}-m^{2} \beta^{2}\right)^{1 / 2} e^{-n x} \mathrm{~d} x$.

After evaluating the above integrals, we get

$I_{1}^{\prime}=\sum_{n=1}^{\infty} \frac{3 m^{2} \beta^{2}}{n^{2}} K_{2}(n m \beta)=3 m^{2} \beta^{2} I(2, n m \beta)$,

$I_{2}^{\prime}=\sum_{n=1}^{\infty} \frac{m^{3} \beta^{3}}{n} K_{1}(n m \beta)=m^{3} \beta^{3} I(1, n m \beta)$,

where

$$
I(\gamma, Z)=\sum_{n=1}^{\infty} \frac{K_{\gamma}(n Z)}{n^{\gamma}} .
$$

The convergent series $I(\gamma, Z)$ has been evaluated by Braden [18]. We just state here the asymptotic behaviour of $I(1, Z)$ and $I(2, Z)$ when $\beta \rightarrow 0$ :

$$
\begin{aligned}
& I(1, Z) \sim \frac{\pi^{2}}{6 Z}-\frac{\pi}{2}, \\
& I(2, Z) \sim \frac{\pi^{4}}{45 Z^{2}}-\frac{\pi^{2}}{2} .
\end{aligned}
$$

Using the results in (3.11) we get

$$
E_{\mathrm{glu}}=\frac{g_{\mathrm{glu}}}{2 \pi^{2}}\left[\frac{(\pi T)^{4}}{15}-\frac{m^{2}}{12}(\pi T)^{2}\right] .
$$

Also $E_{\mathrm{vac}}=B$.
Now, if we include the quark-quark and gluongluon interaction following [1 k] and reference therein, we get upto first order in $\alpha_{\mathrm{S}}$ the following expression for energy density

$$
\begin{aligned}
E=\frac{6}{\pi^{2}}\left[( 1 - \frac { 2 \alpha _ { \mathrm { S } } } { \pi } ) \left[\frac{1}{4}\left(\frac{\mu}{3}\right)\left(\left(\frac{\mu}{3}\right)^{2}-m_{\alpha}^{2}\right)^{3 / 2}\right.\right. \\
+\frac{m_{\alpha}^{2}}{8}\left(\left(\frac{\mu}{3}\right)\left(\frac{\mu}{3}\right)^{2}-m_{\alpha}^{2}\right)^{1 / 2} \\
\quad-\frac{m_{\alpha}^{4}}{8} \ln \left|\frac{\left(\frac{\mu}{3}\right)\left(\left(\frac{\mu}{3}\right)^{2}-m_{\alpha}^{2}\right)^{1 / 2}}{m_{\alpha}}\right| \\
\left.\left.+\left(\frac{1}{2}\left(\frac{\mu}{3}\right)^{2}-\frac{m_{\alpha}^{2}}{12}\right) \theta^{2}\right]+\left(1-\frac{50 \alpha_{\mathrm{s}}}{21 \pi}\right) \frac{7}{60} \theta^{4}\right] \\
+\frac{8}{15 \pi^{2}}\left(\theta^{4}-\frac{5}{4} m^{2} \theta^{2}\right)\left(1-\frac{15 \alpha_{\mathrm{S}}}{4 \pi}\right)+B,
\end{aligned}
$$

where $\theta=\pi T$ and we have taken $g_{\mathrm{qu}}=12$ and $g_{\mathrm{glu}}=16$. Expressions for the pressure and entropy density are obtained as

$$
\begin{aligned}
P=\ln Z= & \frac{2}{\pi^{2}}\left[( 1 - \frac { 2 \alpha _ { \mathrm { S } } } { \pi } ) \left[\frac{1}{4}\left(\frac{\mu}{3}\right)\left(\left(\frac{\mu}{3}\right)^{2}-m_{\alpha}^{2}\right)^{3 / 2}\right.\right. \\
& +\frac{m_{\alpha}^{2}}{8}\left(\frac{\mu}{3}\right)\left(\left(\frac{\mu}{3}\right)^{2}-m_{\alpha}^{2}\right)^{1 / 2} \\
& -\frac{m_{\alpha}^{4}}{8} \ln \left|\frac{\left(\frac{\mu}{3}\right)+\left(\left(\frac{\mu}{3}\right)^{2}-m_{\alpha}^{2}\right)^{1 / 2}}{m_{\alpha}}\right| \\
& \left.\left.+\left(\frac{1}{2}\left(\frac{\mu}{3}\right)^{2}-\frac{m_{\alpha}^{2}}{12}\right) \theta^{2}\right]+\left(1-\frac{50 \alpha_{\mathrm{S}}}{21 \pi}\right) \frac{7}{60} \theta^{4}\right] \\
& +\frac{8}{45 \pi^{2}}\left(\theta^{4}-\frac{5}{4} m^{2} \theta^{2}\right)\left(1-\frac{15 \alpha_{\mathrm{S}}}{4 \pi}\right)-B \\
= & \frac{1}{3}(E-4 B),
\end{aligned}
$$

where $\ln Z=[\ln Z]_{\mathrm{qu}}+[\ln Z]_{\mathrm{glu}}+[\ln Z]_{\mathrm{vac}}$, and

$$
\begin{aligned}
S= & \frac{2}{\pi}\left(1-\frac{2 \alpha_{\mathrm{S}}}{\pi}\right)\left[\left(\frac{\mu}{3}\right)^{2}-\frac{m_{\alpha}^{2}}{6}\right] \theta+\frac{14}{15 \pi}\left(1-\frac{50}{21} \frac{\alpha_{\mathrm{S}}}{\pi}\right) \theta^{3} \\
& +\frac{32}{45 \pi}\left(\theta^{3}-\frac{5}{8} m^{2} \theta\right) .
\end{aligned}
$$

(3.17) For $P=0$ we have $E=4 B$. 
Hence we get

$$
\begin{aligned}
B=\frac{2}{\pi^{2}}[ & \left(1-\frac{2 \alpha_{\mathrm{S}}}{\pi}\right)\left[\frac{1}{4}\left(\frac{\mu}{3}\right)\left(\left(\frac{\mu}{3}\right)^{2}-m_{\alpha}^{2}\right)^{3 / 2}\right. \\
& +\frac{m_{\alpha}^{2}}{8}\left(\frac{\mu}{3}\right)\left(\left(\frac{\mu}{3}\right)^{2}-m_{\alpha}^{2}\right)^{1 / 2} \\
& -\frac{m_{\alpha}^{2}}{8} \ln \frac{\left(\frac{\mu}{3}\right)+\left(\left(\frac{\mu}{3}\right)^{2}-m_{\alpha}^{2}\right)^{1 / 2}}{m_{\alpha}} \\
& \left.+\left(\frac{1}{2}\left(\frac{\mu}{3}\right)^{2}-\frac{m_{\alpha}^{2}}{12}\right) \theta^{2}\right] \\
& \left.+\left(1-\frac{50}{21} \frac{\alpha_{\mathrm{S}}}{\pi}\right) \frac{7}{60} \theta^{4}\right] \\
& +\frac{8}{45} \pi^{2}\left(1-\frac{15}{4} \frac{\alpha_{\mathrm{S}}}{\pi}\right)\left(\theta^{4}-\frac{5}{4} m^{2} \theta^{2}\right) .
\end{aligned}
$$

It is to be noted that the temperature independent terms of the energy density expression at $T \neq 0$ are identical with that of the free energy density expression at $T=0$ (Ref. (2.7)).

A few words about the value of $\alpha_{s}$ in (3.21). To compare our results with those of Rafelski et al. [1 k] we took $\alpha_{S}=0.5$, but the effect of finite quark mass would necessitate a change in the value of $\alpha_{s}$. The choice of $\alpha_{\mathrm{S}}=0.5$ is motivated by fits of charmonium and ypsilonium. But a much smaller value of $\alpha_{\mathrm{s}}=0.2$ is found in the time like region of momentum transfer in $\mathrm{e}^{+} \mathrm{e}^{-} \rightarrow$ hadrons experiments. In presenting numerical calculations, we have considered both these values of $\alpha_{\mathrm{S}}$, to show the change of $T_{\mathrm{c}}, \mu_{\mathrm{c}}, E$ with $\alpha_{\mathrm{S}}$.

Again from (3.18), (3.19), (3.20), (3.21) it is clear that for $m_{\alpha}=0, m=0$ our results are identical to those obtained by Rafelski [ $1 \mathrm{k}]$. But the advantage here is that one can find the various thermodynamical quantities taking quark-gluon masses in accout. So in that sense the formulae are far more general. Using (3.21), one can draw the critical curve in the $\mu-T$ plane. In Figure 1 the critical curve for the quark-gluon plasma has been drawn for $m_{\alpha}=0$ and $m_{\alpha}=1$ and 5 ( $m=0$ in both cases), $\alpha_{\mathrm{S}}=0.5$ and $\alpha_{\mathrm{S}}=0.2$. All the quantities have been normalised to $B^{1 / 4}$. The critical density can be calculated from (3.21) by taking $T=0$. In Figure 2, $\mu_{\mathrm{c}}$ has been plotted against $m_{\alpha}$. Since all the quantities have been calculated in terms of $B^{1 / 4}$, we do not show here the variation of $\mu_{\mathrm{c}}$ with $B^{1 / 4}$, which is implicit in our calculation. In Figure 2 we have not indicated $T_{\mathrm{c}}$, as a separate calculation has been done when $\mu=0$. This is discussed in Section 4.

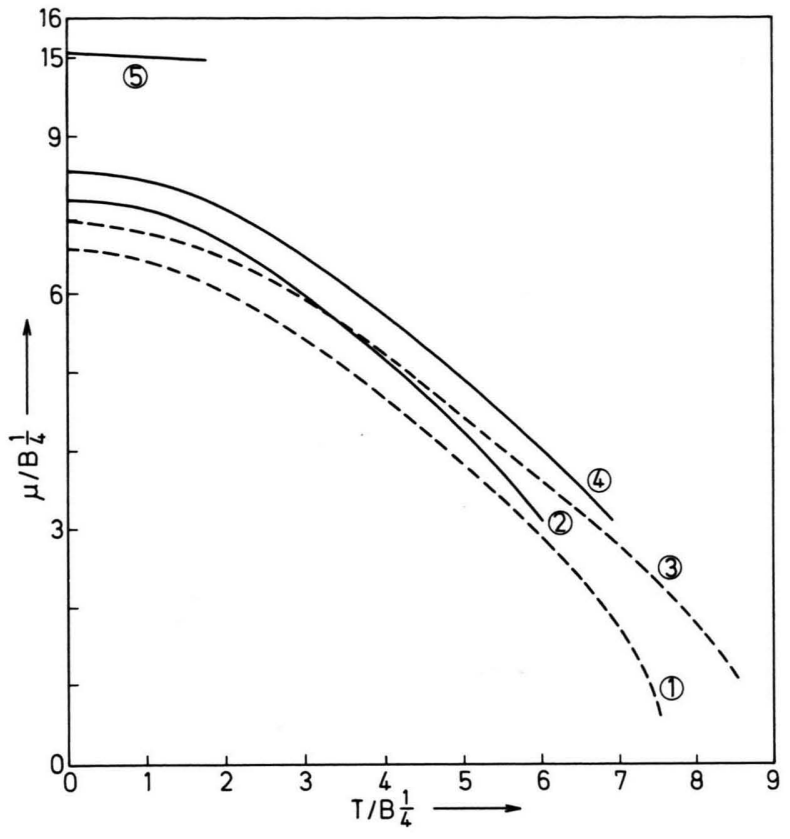

(1) $m_{\alpha}=0, \alpha_{\mathrm{S}}=0.2$; (2) $m_{\alpha}=1, \alpha_{\mathrm{S}}=0.2$; (3) $m_{\alpha}=0, \alpha_{\mathrm{S}}=0.5$; (4) $m_{\alpha}=1, \alpha_{s}=0.5$; (5) $m_{\alpha}=5, \alpha_{S}=0.5$.

Fig. 1. Plot of $\mu / B^{1 / 4}$ vs. $T / B^{1 / 4}$.

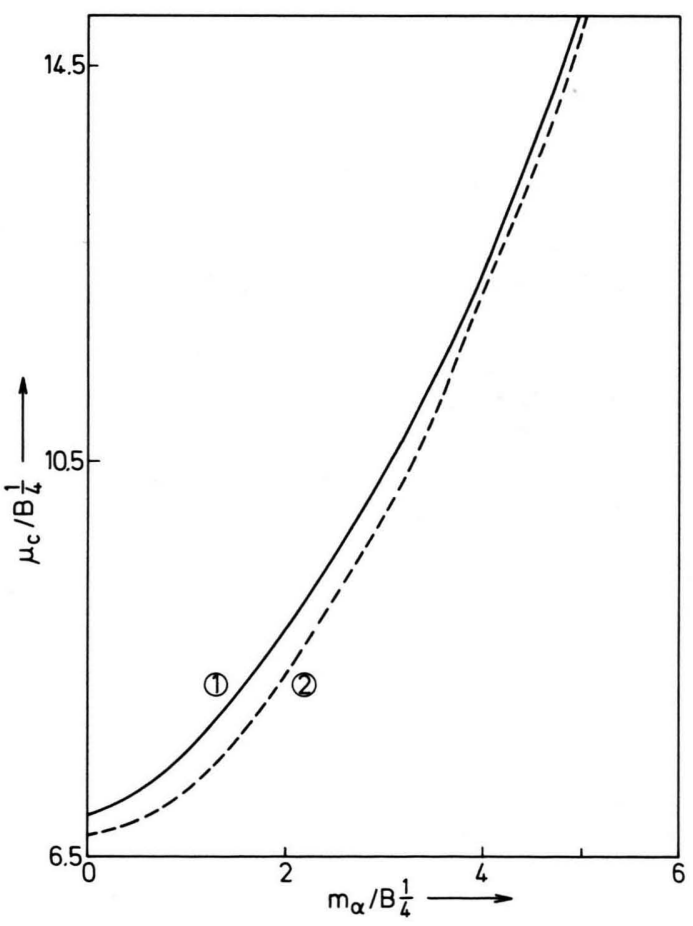

Curve (1) $\alpha_{\mathrm{S}}=0.5$; Curve (2) $\alpha_{\mathrm{S}}=0.2$

Fig. 2. Plot of $\mu_{\mathrm{c}} / B^{1 / 4}$ vs. $m_{\alpha} / \mathrm{B}^{1 / 4}$. 


\section{Calculation of Critical Temperature}

In order to obtain the effect of finite quark mass on the lower bound $T_{\mathrm{c}}$ one should study (3.19) for $P=0$ at $\mu=0$. But since (3.18) has been obtained on the assumption $\mu_{\alpha}>m_{\alpha}$, one cannot use it for calculating the critical temperature. (This will be the point of departure of our method from that of others, specially Rafelski [1 k]). We believe that $(3.18)$ has been obtained assuming a not too small $\mu_{\alpha}$ and to obtain correct formulae for $\mu_{\alpha}=0$ one should start ab initio.

Starting from the partition function for $\mu=0$, the energy density for free quarks can be written as

$$
\begin{aligned}
E_{\mathrm{qu}}= & \frac{g_{\mathrm{qu}}}{2 \pi^{2}} \int_{m_{\alpha}}^{\alpha} \varepsilon_{\alpha}^{2}\left(\varepsilon_{\alpha}^{2}-m_{\alpha}^{2}\right)^{1 / 2} \frac{e^{-\varepsilon_{\alpha} \beta} \mathrm{d} \varepsilon_{\alpha}}{1+\exp \left(-\varepsilon_{\alpha} \beta\right)} \\
= & \frac{g_{\mathrm{qu}}}{2 \pi^{2} \beta^{4}} \sum_{n=1}^{\alpha}(-1)^{n} \int_{m_{\alpha} \beta}^{\alpha} x^{2}\left(x^{2}\right. \\
& \left.-m_{\alpha}^{2} \beta^{2}\right)^{1 / 2} e^{-(n+1) x} \mathrm{~d} x,
\end{aligned}
$$

where $x=\varepsilon_{\alpha} \beta$

$$
\begin{array}{r}
=\frac{g_{\mathrm{qu}}}{2 \pi^{2} \beta^{4}} \sum_{n=1}^{\infty}(-1)^{n}\left[\frac{3 m_{\alpha}^{2} \beta^{2}}{(n+1)^{2}} K_{2}(n+1) m_{\alpha} \beta\right. \\
\left.+\frac{m_{\alpha}^{3} \beta^{3}}{(n+1)} K_{1}(n+1) m_{\alpha} \beta\right] .
\end{array}
$$

Again, using Braden's [17] method we get

$$
E_{\mathrm{qu}}=\frac{g_{\mathrm{qu}}}{2 \pi^{2}}\left(\frac{7}{120} \theta^{4}-\frac{m_{\alpha}^{2}}{24} \theta^{2}\right) \text {. }
$$

Equation (3.18) becomes, after due correction due to quark-quark and gluon-gluon interaction,

$$
\begin{aligned}
E= & \frac{6}{\pi^{2}}\left[\left(1-\frac{50}{21} \frac{\alpha_{\mathrm{s}}}{\pi}\right)\left(\frac{7}{120} \theta^{4}-\frac{m_{\alpha}^{2}}{24} \theta^{2}\right)\right] \\
& +\frac{8}{15 \pi^{2}}\left(1-\frac{15}{4} \frac{\alpha_{\mathrm{s}}}{\pi}\right)\left(\theta^{4}-\frac{5}{4} m^{2} \theta^{2}\right)+B
\end{aligned}
$$

with

$$
\begin{aligned}
B= & \frac{2}{\pi^{2}}\left(1-\frac{50}{21} \frac{\alpha_{\mathrm{S}}}{\pi}\right)\left(\frac{7}{120} \theta^{4}-\frac{m_{\alpha}^{2}}{24} \theta^{2}\right) \\
& +\frac{8}{45 \pi^{2}}\left(1-\frac{15}{4} \frac{\alpha_{\mathrm{s}}}{\pi}\right)\left(\theta^{4}-\frac{5}{4} m^{2} \theta^{2}\right) .
\end{aligned}
$$

Comparing our result (4.3) for $m_{\alpha}=0$ with that of Rafelski [1 k] we see that there is a difference of a factor of 2 between the two results. But our result is consistent with that of Satz [1 j], and we think it is the correct result for $\mu=0$.

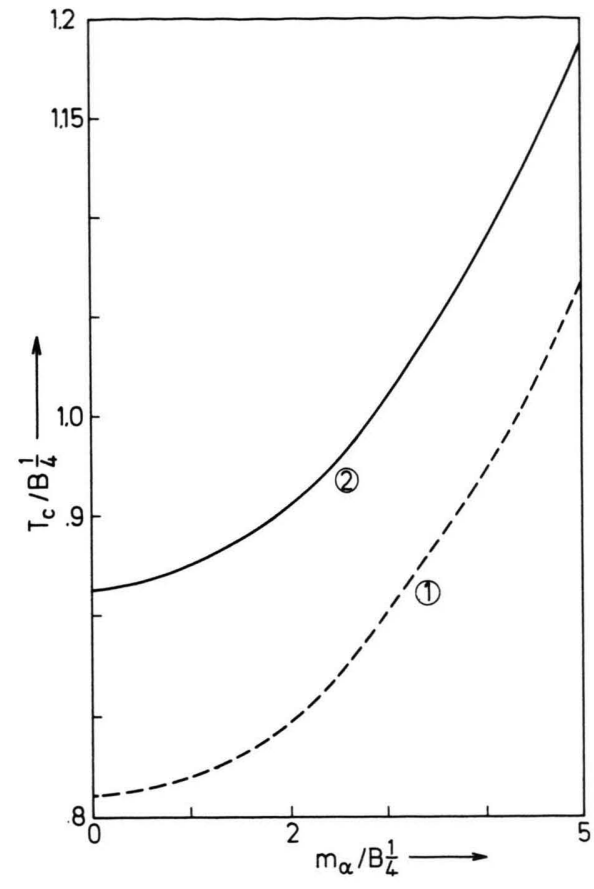

Curve (1) $\alpha_{\mathrm{S}}=0.2$; Curve (2) $\alpha_{\mathrm{S}}=0.5$

Fig. 3. Plot of $T_{\mathrm{c}} / B^{1 / 4}$ vs. $m_{\alpha} / B^{1 / 4}$.

The critical temperature can be obtained from putting $P=0$. Using (4.3) for the energy density we get (for $m_{\alpha}=0$ ) $T_{\mathrm{c}} \sim 0.91566 B^{1 / 4} \simeq 173.97 \mathrm{MeV}$. However, if we take $m_{\alpha} \sim B^{1 / 4}$, then $T_{\mathrm{c}} \sim 0.9256 B^{1 / 4} \simeq$ $175.86 \mathrm{MeV}$. (We have taken $\alpha_{\mathrm{S}}=0.5$ and $B=190$ $\mathrm{MeV}$.) It should be mentioned here that $m_{\alpha} \sim B^{1 / 4}$ $=200 \mathrm{MeV}$ is not a realistic quark mass for the study of the quark-gluon plasma. Hence we have shown the effect of $m_{\alpha}$ on $T_{\mathrm{c}}$ by plotting $T_{\mathrm{c}}$ against $m_{\alpha}$ in Figure 3. But as mentioned before, the effect of introducing finite quark mass would also necessitate a change in the values of $\alpha_{\mathrm{s}}$ and $B$. Hence we have plotted $T_{\mathrm{c}}$ against $m_{\alpha}$ for two different values of $\alpha_{S}$, viz. 0.5 and 0.2 . As before, $T_{\mathrm{c}}$ and $m_{\alpha}$ are normalised by $B^{1 / 4}$, whereby their variation with respect to $B^{1 / 4}$ is implicit in our plot.

\section{Phase Transition and Energy Gap}

The phase transition from the hadronic gas phase to the quark-gluon plasma is mainly determined by the energy density of the fire ball. A major topic of contemporary research is about the order of transition. Since the main difference of our work from others 


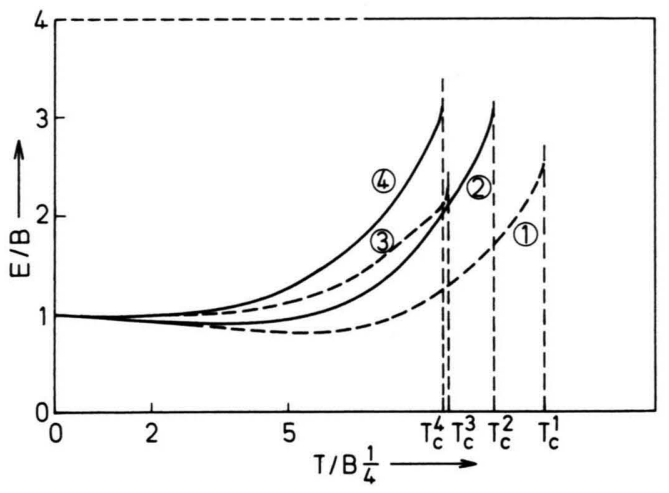

(1) $m_{\alpha}=1, \alpha_{\mathrm{S}}=0.5$; (2) $m_{\alpha}=1, \alpha_{\mathrm{S}}=0.2$; (3) $m_{\alpha}=0, \alpha_{\mathrm{S}}=0.5$; (4) $m_{\alpha}=0, \alpha_{\mathrm{s}}=0.2$

Fig. 4. Plot of $E / B$ vs. $T / B^{1 / 4}$.

([1 k] and references therein) lies in taking into accounts the effect of finite quark mass on quantities of interest in quark-gluon plasma studies, the basic conclusion would be the same as that of others. This means that it is likely that the phase transition is a first order phase transition, though it cannot be determined unambiguously. A detailed study is outside the scope of this work, which has rather the humble aim of presenting some relevant calculations. However, it may be of some interest to show the energy density aygainst $T$. In Figure 4, we have drawn the energy density against $T$ for values of $m_{\alpha}=0$ and $m_{\alpha}=1$ (normalised to $B^{1 / 4}$ ).

[1] (a) Ya B. Zeldovich, JETP 37, 569 (1959). - (b) N. Itoh, Prog. Theor. Phys. 44, 291 (1970). - (c) G. Chapline and M. Nauenberg, Nature 264, 235 (1976) and Phys. Rev. D 16, 450 (1977). - (d) B. A. Freedom and L. D. McLerran, Phys. Rev. D 16, 1169 (1977). - (e) S. A. Chin, Phys. Lett. 78 B, 552 (1978). - (f) J. I. Kapusta, Nucl. Phys. B 148, 461 (1979). - (g) J. Rafelski and R. Hagedorn, From Hadron Gas to Quark Matter, II, in: Thermodynamicals of Quarks and Hadrons (H. Satz, ed.), North Holland, Amsterdam 1981. - (i) E. V. Shuryak, Phys. Lett. 81 B, 65 (1979) and Phys. Rep. 61, 71 (1980). (j) H. Satz, Phys. Rep. 88, 349 (1982). - (k) J. Rafelski, Phys. Rep. 88, 331 (1982). - (l) S. Kagiyama, S. Hirooka, H. Kikukawa, J. Kikukawa, and Y. Nishiyama, Prog. Theor. Phys. 62, 490 (1979); 63, 956 (1980); 65, 1684 (1981). - (m) B. Svetitsky, Nucl. Phys. A 461, 71 (1987) and references therein.

[2] H. Satz, Proc. 5th High Energy Heavy Ion Study, Berkley, California 1981, and references therein.

[3] (a) A. M. Polyakov, Phys. Lett. 72 B, 477 (1978). (b) L. Susskind, Phys. Rev. D 20, 2610 (1979).

[4] L. D. McLerran and B. Svetitsky, Phys. Lett. 98 B, 195 (1981) and Phys. Rev. D 24, 450 (1981).

[5] J. Kuti, J. Polonyi, and K. Szlachanyi, Phys. Lett. 101 B, 89 (1981).

\section{Discussion}

In this paper we have obtained analytical formulae for various thermodynamical quantities like energy density, pressure, entropy density for the quark-gluon plasma without neglecting quark and gluon masses.

We have also calculated the effect of finite quark mass on these quantities numerically to highlight the difference from the massless case. The critical temperature is obtained from (4.4). The effect of finite quark masses on the critical curve in the $\mu-T$ plane has been shown explicitly for different quark masses with different coupling constant $\alpha_{\mathrm{S}}$ (viz., $\alpha_{\mathrm{S}}=0.5$ and $\alpha_{\mathrm{S}}=0.2$ ). The variation of the critical temperature $T_{\mathrm{c}}$ and critical chemical potential $\mu_{\mathrm{c}}$ with quark masses for different $\alpha_{S}$ have also been calculated.

As mentioned earlier, one of the major topics of interest is the order of the phase transition in the quark-gluon plasma. Since our calculation differs basically from those of others $([1 \mathrm{k}]$ and references therein) in the fact that we have taken into account of finite quark mass, one sees that qualitatively the picture will not change much. Though the physical arguments are in favour of a first order transition, it is not yet decided unambiguously. However, we have plotted the energy gap with temperature for $m_{\alpha}=0$ and $m_{\alpha}=1$ (in units of $B^{1 / 4}$ ) for values of $\alpha_{S}=0.2$ and 0.5 . It is seen that qualitatively the energy gap does not change much. Finally, we hope that our calculations will be of use to find out the effect of finite quark masses on the thermodynamical properties of quark-gluon plasmas.

[6] J. Engles, F. Karsch, I. Montvay, and H. Satz, Phys. Lett. 101 B, 89 (1981); Phys. Lett. 102 B, 332 (1981).

[7] H. Satz, Ann. Rev. Nucl. Part-Sci. 35, 245 (1985).

[8] J. Cleymans, R. Gavai, and E. Suhonen, Phys. Rep. 130, 210 (1986).

[9] B. Svetitsky, Phys. Rep. 132, 1 (1986).

[10] D. E. Miller and K. Redlich, Phys. Rev. D 37, 3716 (1988), and references therein.

[11] A. Chodos, R. L. Jaffe, K. Johnson, C. B. Thorn, and V. F. Weisskopf, Phys. Rev. D 9, 3471 (1974).

[12] T. De Grand, R. L. Jaffe, K. Johnson, and J. Kiskis, Phys. Rev. D 12, 2060 (1975).

[13] W. A. Barden, M. S. Chanowitz, S. D. Drell, M. Weinstein, and T. M. Yan, Phys. Rev. D 11, 1094 (1975).

[14] P. Gnadig, P. Hasentartz, J. Kuti, and A. S. Sazalay, Phys. Lett. 64 B, 62 (1976).

[15] R. Freidberg and T. D. Lee, Phys. Rev. D 16, 1096 (1977).

[16] S. Kagiyama, S. Hirooka, H. Kikukawa, and J. Kikukawa, Prog. Theor. Phys. 69, 579 (1983).

[17] R. K. Pathria, in: Statistical Mechanics, Pergamon Press, International Series in Natural Philosophy, Vol. 45 (1986), reprint.

[18] H. W. Braden, Phys. Rev. D 25, 1028 (1982). 